\title{
Highway Traffic Flow Nonlinear Character Analysis and Prediction
}

\author{
Meng Hui, Lin Bai, YanBo Li, and QiSheng Wu \\ School of Electronics and Control, Chang'an University, Xian, Shaanxi 710064, China \\ Correspondence should be addressed to Meng Hui; xmwswws@163.com
}

Received 23 June 2014; Revised 12 September 2014; Accepted 18 September 2014

Academic Editor: Shouming Zhong

Copyright ( $) 2015$ Meng Hui et al. This is an open access article distributed under the Creative Commons Attribution License, which permits unrestricted use, distribution, and reproduction in any medium, provided the original work is properly cited.

\begin{abstract}
In order to meet the highway guidance demand, this work studies the short-term traffic flow prediction method of highway. The $\mathrm{Yu}-\mathrm{Wu}$ highway which is the main road in Chongqing, China, traffic flow time series is taken as the study object. It uses phase space reconstruction theory and Lyapunov exponent to analyze the nonlinear character of traffic flow. A new Volterra prediction method based on model order reduction via quadratic-linear systems (QLMOR) is applied to predict the traffic flow. Compared with Taylor-expansion-based methods, these QLMOR-reduced Volterra models retain more information of the system and more accuracy. The simulation results using this new Volterra model to predict short time traffic flow reveal that the accuracy of chaotic traffic flow prediction is enough for highway guidance and could be a new reference for intelligent highway management.
\end{abstract}

\section{Introduction}

The prediction of highway traffic flow is a crucial part of transportation planning, traffic control, and intelligent transportation systems [1]. In particular, short-term traffic flow predicting can provide real time information for highway guidance. Also accurate predictions of traffic flow are essential part of efficient operations in advanced traffic management systems. In recent years, the short-term predicting technologies have gotten the attention of many researchers.

Short-term traffic flow is a typical nonlinear time series in a time horizon of $15 \mathrm{~min}$ or less [2]. During the decades a variety of techniques have been applied to forecast the shortterm traffic flow, such as fuzzy theory [3], neural networks [4], Kalman filter [5], and wavelet analysis [6] et al. But the models generated by these methods could not capture some strongly nonlinear characteristics of short-term traffic flow data. The prediction results may have big error. Chaos theory is an effective tool to study nonlinear system. This tool has been applied to forecast short-term traffic flow time series $[7,8]$. The forecasting results are better than other methods applied before. However, the accuracy of chaotic forecasting result is not enough for real time highway guidance. There remains room for proposing new prediction technology to make the prediction more accurate.

To use chaos theory we should judge whether the traffic flow data is a chaotic time series or not. The largest Lyapunov exponent is usually used to achieve this goal. Wolf algorithm [9], Jacobin algorithm [10], and small data method [11] are three algorithms often used to calculate Lyapunov exponent. If the traffic flow data is a chaotic time series, the phase space reconstruction theory could be applied to predict the traffic flow.

In this paper, the traffic flow data of Yu-Wu highway in Chongqing, China, is taken as study object. We study the general day traffic flow character of highway and the holiday traffic flow model is not contained in this paper. Firstly, the approved small data method is used to judge whether the traffic flow data is a chaotic time series. Secondly, phase space reconstruction theory is used to reconstruct the traffic flow system. Thirdly, a new predicting model based on model order reduction via quadratic-linear systems is used to predict the chaotic traffic flow. This new prediction model has no truncation error and contains more information of original system. It is more accurate than other prediction models ever used before. 


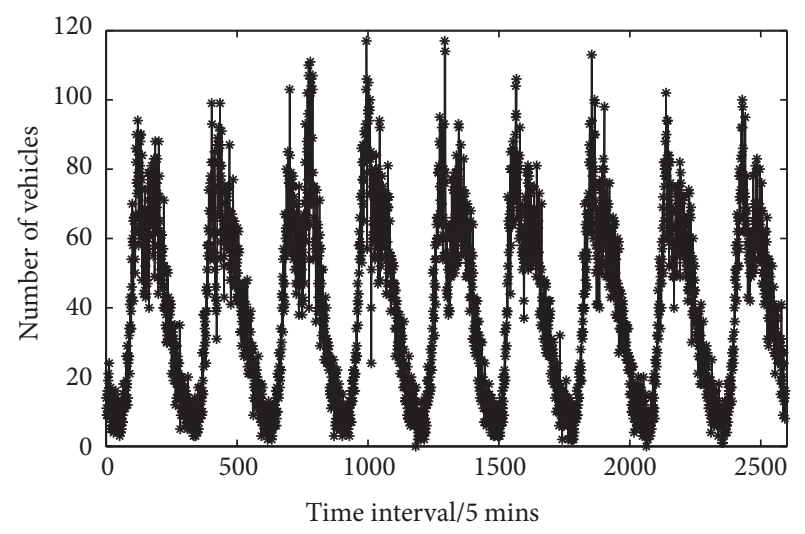

Figure 1: Traffic flow of Yu-Wu highway.

\section{Traffic Flow Data Phase Space Reconstruction}

2.1. Traffic Flow Data. All the traffic flow data are collected at Yu-Wu highway in Chongqing, China. Sampling time begins at 0:00 November 20,2013, and ends at 0:00 November 27, 2013. Time interval is 5 minutes. Figure 1 shows the traffic data.

2.2. Mutual Information Method. Takens theorem [12] is the mathematical base of time series phase space reconstruction. Choosing optimal time delay $\tau$ and embedding dimension $d$ is the key of phase space reconstruction. Mutual information method is an effective tool to calculate $\tau$.

Assuming chaotic time series $r_{1}, r_{2}, \ldots$ time delay is $\tau$ and embedding dimension is $d$. The reconstruction phase space $Q$ of this time series is

$$
Q(t)=\left(q_{0}(t), q_{1}(t), \ldots, q_{n}(t)\right)
$$

where $q_{n}(t)=r(t+n \tau)$. The average information of variable $r$ is called information entropy which can be described as

$$
H(R)=-\sum_{i} P_{r}\left(r_{i}\right) \log P_{r}\left(r_{i}\right)
$$

where $P_{r}\left(r_{i}\right)$ is the probability of event $r$ in $i$. Let $[r, u]=$ $[q(t), q(t+\tau)]$, if system $(R, U)$ is a coupling system and $r$ is given as $r_{i}$ the uncertainty of $u$ is

$$
\begin{aligned}
H\left(U \mid r_{i}\right) & =-\sum_{j} P_{u \mid r}\left(u_{j} \mid r_{i}\right) \log \left[P_{u \mid r}\left(u_{j} \mid r_{i}\right)\right] \\
& =-\sum_{i} \frac{P_{r u}\left(r_{i}, u_{i}\right)}{P_{r}\left(r_{i}\right)} \times \log \left[\frac{P_{r u}\left(r_{i}, u_{i}\right)}{P_{r}\left(r_{i}\right)}\right],
\end{aligned}
$$

where $P_{u \mid r}\left(u_{j} \mid r_{i}\right)$ is the probability that a measurement of $u$ yields $u_{j}$, given that the measured value of $r$ is $r_{i}$. Given that

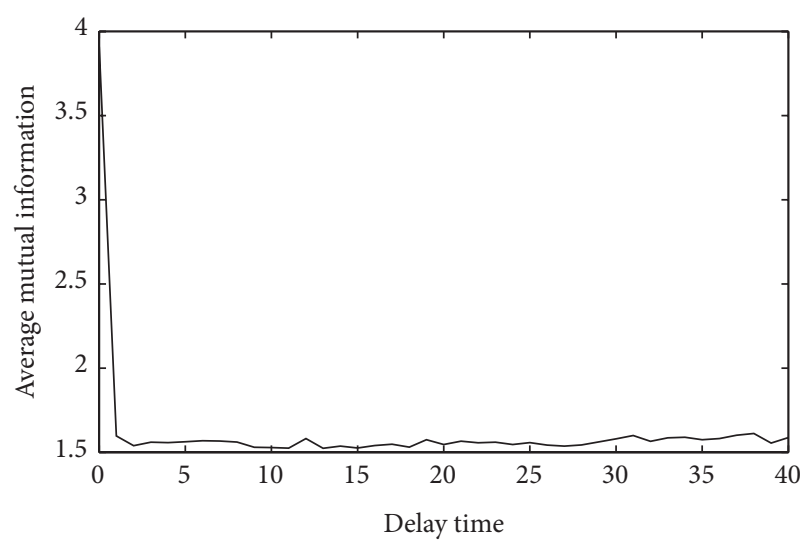

FIGURE 2: Mutual information method calculates delay time.

$q$ has been measured at time $t$ the average uncertainty in a measurement of $r$ at time $t+\tau$ is

$$
\begin{gathered}
H(U \mid R)=\sum_{i} P_{r}\left(r_{i}\right) H\left(U \mid r_{i}\right)=H(R, U)-H(R) \\
H(R, U)=-\sum_{i, j} P_{r u}\left(r_{i}, u_{i}\right) \log P_{r u}\left(r_{i}, u_{i}\right),
\end{gathered}
$$

where $H(R, U)$ is the uncertainty of $u$ in isolation. $H(U \mid R)$ is the uncertainty of $u$ in the measurement of $r$. The amount of a measurement of $r$ reduces the uncertainty of $u$. The mutual information is

$$
\begin{aligned}
I(U, R) & =H(U)-H(U \mid R) \\
& =H(Q)+H(R)-H(R, U)=I(R, U) .
\end{aligned}
$$

The mutual information is a function of $P_{r u}$ which is the joint distribution probability of the event $r$ and $u$. If a vector is a phase space reconstruction while the mutual information $I$ first reaches the minimum the time delay could be the delay time of phase space reconstruction.

Figure 2 shows the traffic flow delay time calculated by the mutual information method. When $\tau=2$ the system reaches the minimum so the optimal delay time $\tau=2$.

2.3. CAO Method. Time series $y$ can be reconstructed in phase space as follows: $Y(i)=\{y(i), y(i+\tau), \ldots, y(i+(d-$ $1) \tau)\}$, where $\tau$ is the delay time and $d$ is the embedding dimension. The nearest neighbor point of $i$ is $Y^{N N}(i)$; the distance between two points is

$$
\begin{aligned}
L_{d}^{2}(i) & =\left\|Y(i)-Y^{N N}(i)\right\|^{2} \\
& =\sum_{j=1}^{d}\left\{y[i+(j-1) \tau]-y^{N N}[i+(j-1) \tau]\right\}^{2} .
\end{aligned}
$$


When the space dimension changes from $d$ to $d+1$, the distance between two points is

$$
\begin{aligned}
L_{d+1}^{2}(i) & =\sum_{j=1}^{d+1}\left\{y[i+(j-1) \tau]-y^{N N}[i+(j-1) \tau]\right\}^{2} \\
& =L_{d}^{2}(i)+\left|y[i+\tau d]-y^{N N}[i+\tau d]\right|^{2} .
\end{aligned}
$$

Define

$$
a_{1}(i, d)=\frac{\left|y[i+\tau d]-y^{N N}[i+\tau d]\right|}{L_{d}(i)}
$$

If $a_{1}(i, d)>R_{\tau}, R_{\tau} \in[10,50], Y^{N N}(i)$ is the false nearest neighbor point. Substitute (6) into (8); we can get

$$
a_{1}(i, d)=\frac{y[i+\tau d]-y^{N N}[i+\tau d]}{\left\|Y(i)-Y^{N N}(i)\right\|} .
$$

CAO [13] made some changes to (9) yielding

$$
a_{2}(i, d)=\frac{\left\|Y_{d+1}(i)-Y_{d+1}^{N N}(i)\right\|}{\left\|Y_{d}(i)-Y_{d}^{N N}(i)\right\|} .
$$

Here $\|\cdot\|$ is the Euclidian distance. Let $E(d)=1 /(N-$ $d \tau) \sum_{i=1}^{N-d \tau} a_{2}(i, d), E_{1}(d)=E(d+1) / E(d) ; E(d)$ is only dependent on the dimension $d$ and delay time $\tau$. It is found that $E_{1}(d)$ stops changing when $d$ is greater than $d_{0} . d_{0}$ is the optimal embedding dimension of reconstruction space.

For time series data from a random set of numbers, $E_{1}(d)$ will never attain a saturation value as $d$ increases in principle. But in practical computations, it is difficult to resolve whether $E_{1}(d)$ is slowly increasing or has stopped changing if $d$ is sufficiently large. In fact, since available observed data samples are limited, it may happen that $E_{1}(d)$ stops changing at some dimension although the time series is random. Therefore, we define

$$
\begin{gathered}
E^{\prime}(d)=\frac{1}{N-d \tau} \sum_{i=1}^{N-d \tau}\left|y[i+\tau d]-y^{N N}[i+\tau d]\right| \\
E_{2}(d)=\frac{E^{\prime}(d+1)}{E^{\prime}(d)} .
\end{gathered}
$$

For random series, $E_{2}(d)=1$. For deterministic series, there must exist $d$ making $E_{2}(d) \neq 1$.

Figure 3 shows the embedding dimension calculated by CAO method. The optimal embedding dimension $d=10$.

\section{Chaotic Character of Traffic Flow}

Correlation dimension and largest Lyapunov exponent are two main indexes to distinguish chaotic system from others.

3.1. Correlation Dimension. G-P algorithm is the most popular method to calculate correlation dimension for its simplicity. Figure 4 shows the $\ln C(r, m)-\ln r$ curves. These curves got through increasing embedding dimension until the slope

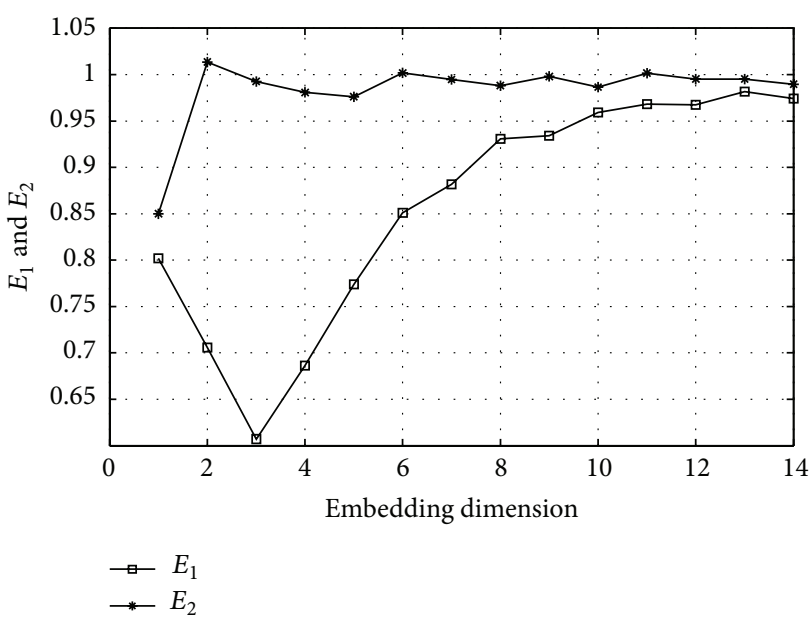

FIGURE 3: Embedding dimension calculated by CAO method.

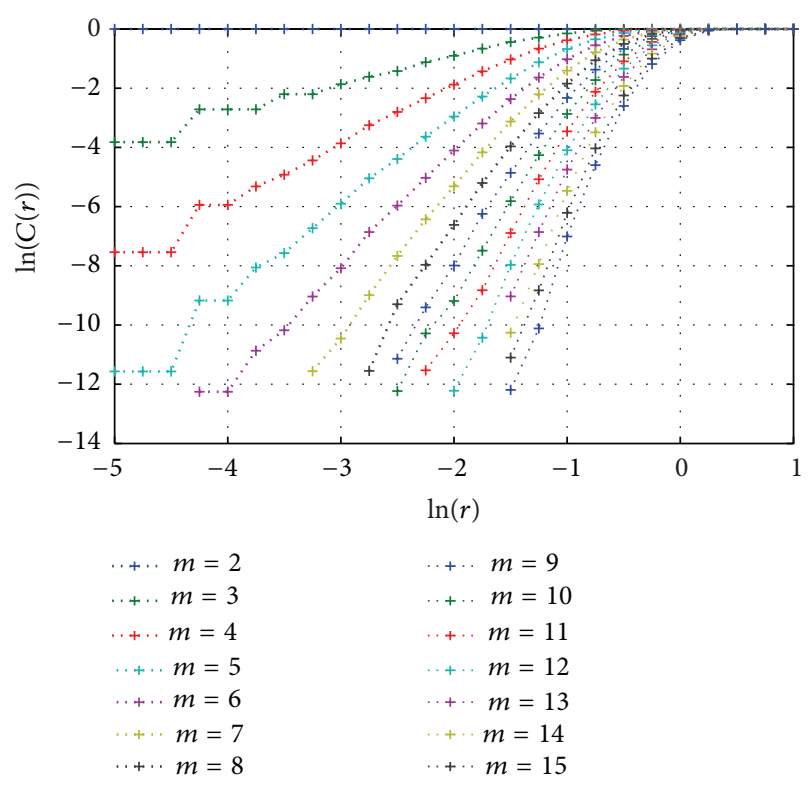

FIGURE 4: Correlation dimension.

of the curve's linear part is almost constant. Then, the correlation dimension estimation can be obtained using least square regression.

Through Figure 4 we can get the correlation dimension $g(m)=5.4116$; its fractal reveals that the system is a chaotic system.

3.2. The Largest Lyapunov Exponent of Traffic Flow. In the reconstructed phase space the distance between each point $X_{j}$ and its nearest neighbor point $X_{j^{\prime}}$ is expressed as

$$
d_{j}(0)=\min _{X_{j}}\left\|X_{j}-X_{j^{\prime}}\right\|, \quad\left|j-j^{\prime}\right|>P,
$$


where $P$ is the mean period. Based on the study in [14] the largest Lyapunov exponent is defined as

$$
\lambda_{1}(i)=\frac{1}{i \Delta t} \cdot \frac{1}{\left(T_{N}-i\right)} \sum_{j=T_{0}}^{T_{N}-i} \ln \frac{d_{j}(i)}{d_{j}(0)},
$$

where $\Delta t$ is sampling period and $d_{j}(i)$ is the distance between the $j$ th pair of nearest neighbors after $i$ discrete-time steps. Sato also gives an alternate form of (13)

$$
\lambda_{1}(i, k)=\frac{1}{k \Delta t} \cdot \frac{1}{\left(T_{N}-k\right)} \sum_{j=T_{0}}^{T_{N}-i} \ln \frac{d_{j}(i+k)}{d_{j}(i)},
$$

where $k$ is constant. Combining (13) and (14) yields

$$
d_{j}(i) \approx C_{j} e^{\lambda_{1}(i \Delta t)}, \quad C_{j}=d_{j}(0) .
$$

By taking the logarithm of both sides of above equation, we obtain

$$
\ln d_{j}(i)=\ln C_{j}+\lambda_{1}(i \Delta t) .
$$

The largest Lyapunov exponent equals the slope of (16).

We can obtain the largest Lyapunov exponent of traffic flow data $\lambda=0.1760$. Its a positive value which means the system exist chaos phenomena.

\section{The Volterra Prediction Model}

For single-input-multiple-output system in the form of

$$
\dot{x}=G x+B u,
$$

where $x \in R^{n}$ are the state variables, $u=a \cos (\omega t), \omega \in R$ is the input signal.

Assume an autonomous system as follows:

$$
\dot{v}=S v, \quad S=\left[\begin{array}{cc}
0 & \omega \\
-\omega & 0
\end{array}\right], \quad v(0)=\left[\begin{array}{l}
1 \\
0
\end{array}\right] .
$$

Let $v=\left[v_{1}, v_{2}\right]^{T}, v \in R^{2}$. The system matrix $S$ could be decomposition

$$
S Q=Q \Lambda, \quad \Lambda=\left[\begin{array}{cc}
j \omega & 0 \\
0 & -j \omega
\end{array}\right], \quad Q=\frac{1}{\sqrt{2}}\left[\begin{array}{ll}
1 & j \\
j & 1
\end{array}\right] .
$$

$Q$ is unitary; the close form solution to (18) is

$$
v(t)=\left[\begin{array}{cc}
\cos (\omega t) & \sin (\omega t) \\
-\sin (\omega t) & \cos (\omega t)
\end{array}\right]\left[\begin{array}{l}
1 \\
0
\end{array}\right]=\left[\begin{array}{c}
\cos (\omega t) \\
-\sin (\omega t)
\end{array}\right] .
$$

Also the input $u=a \cos (\omega t)$ can be taken as

$$
u=L v, \quad L=\left[\begin{array}{ll}
a & 0
\end{array}\right] .
$$

Combining (17)-(21) yields

$$
\left[\begin{array}{c}
\dot{x} \\
\dot{v}
\end{array}\right]=\left[\begin{array}{cc}
G & B L \\
0 & S
\end{array}\right]\left[\begin{array}{l}
x \\
v
\end{array}\right] .
$$

If the eigenvalue $\lambda_{i}(G)-\lambda_{j}(S) \neq 0,(i=1,2, \ldots, n, j=1,2)$, the Sylvester equation

$$
G \Pi+B L=\Pi S, \quad \Pi \in R^{n \times 2}
$$

has unique solution. Then let $I_{k}$ denote the $k$ th order identity matrix; we get

$$
\left[\begin{array}{cc}
G & B L \\
0 & S
\end{array}\right]\left[\begin{array}{cc}
I_{n} & \Pi \\
0 & I_{2}
\end{array}\right]=\left[\begin{array}{cc}
I_{n} & \Pi \\
0 & I_{2}
\end{array}\right]\left[\begin{array}{cc}
G & 0 \\
0 & S
\end{array}\right]
$$

from which the solution to (22) is

$$
x(t)-\Pi v(t)=e^{G t}(x(0)-\Pi v(0)) .
$$

Equation (25) shows the system solution dependent on initial condition $x(0)-\Pi v(0)$ and steady state $\Pi v(t)$. If $x(0)=\Pi v(0)$ the system is driven into period steady state regardless of the fact that $G$ is stable or not.

The study [14] reveals that nonlinear ordinary differential equations can be rewritten equivalently in a special representation, quadratic-linear differential algebraic equations (QLDAEs). The QLDAEs take the form as

$$
C \dot{x}=G_{1} x+G_{2} x \otimes x+D_{1} x u+D_{2}(x \otimes x) u+B u .
$$

Similarly, any generalized polynomial system can be converted into generalized QLDAEs as follows:

$$
\begin{aligned}
& \frac{d}{d t}\left[C_{1} x+C_{2} x \otimes x\right] \\
& \quad=G_{1} x+G_{2} x \otimes x+D_{1} x u+D_{2}(x \otimes x) u+B u .
\end{aligned}
$$

The system (27) is equivalent representation of the original system; this step makes no approximation of original system.

Based on the Volterra theory that the response of a nonlinear system can be decomposed into responses of a series of homogeneous nonlinear systems, the solution of system can be written as follows:

$$
x(t)=\sum_{n=1}^{\infty} x_{n}(t)
$$

where $x_{n}(t)=\int_{-\infty}^{+\infty} \cdots \int_{-\infty}^{+\infty} h_{n}\left(\sigma_{1}, \ldots, \sigma_{n}\right) u\left(t-\sigma_{1}\right), \ldots$, and $u\left(t-\sigma_{n}\right) d \sigma_{1}, \ldots, d \sigma_{n} . h_{n}\left(\sigma_{1}, \ldots, \sigma_{n}\right)$ is $n$th order Volterra kernel. If the input of system (17) is $\alpha u$ the response of system is

$$
x(t)=\alpha x_{1}(t)+\alpha^{2} x_{2}(t)+\alpha^{3} x_{3}(t)+\cdots .
$$

Setting $\alpha=1$ and substituting (29) into (26) we can get

$$
\begin{aligned}
C \dot{x}_{1}= & G_{1} x_{1}+B u, \\
C \dot{x}_{2}= & G_{1} x_{2}+G_{2} x_{1} \otimes x_{1}+D_{1} x_{1} u, \\
C \dot{x}_{3}= & G_{1} x_{3}+G_{2}\left(x_{1} \otimes x_{2}+x_{2} \otimes x_{1}\right) \\
& +D_{1} x_{2} u+D_{2}\left(x_{1} \otimes x_{1}\right) u
\end{aligned}
$$

and so on. 


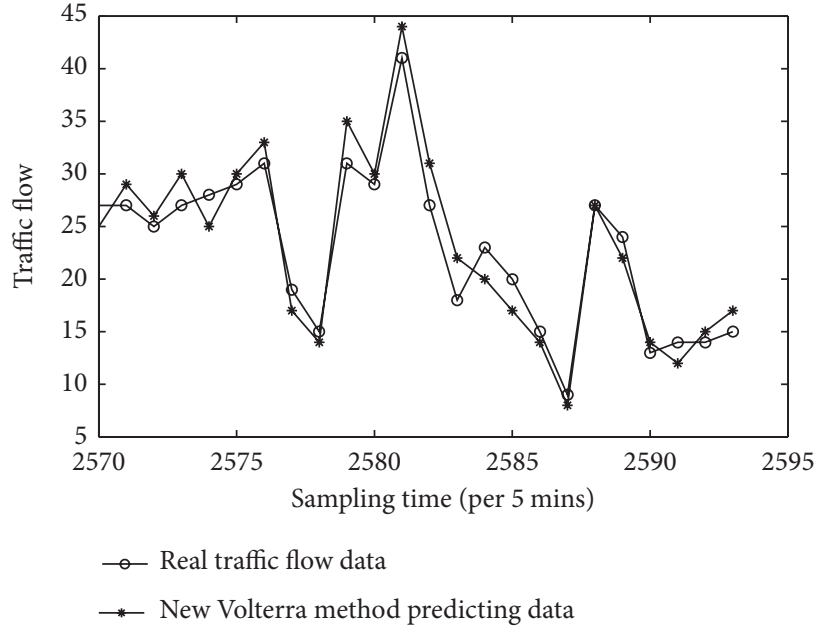

(a)

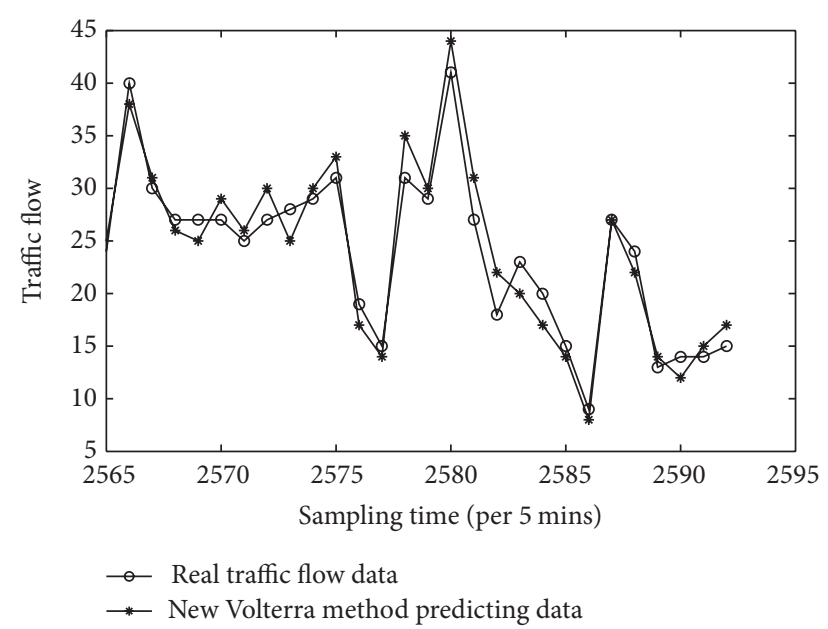

(b)

FIGURE 5: Prediction results of new Volterra algorithm: (a) 2 hours, (b) 4 hours.

Substituting (21) into (30) the steady state solution of system is $x_{1}(t)=\Pi_{1} v(t)$, and $\Pi_{1} \in R^{n \times 2}$ solves the Sylvester equation

$$
G_{1} \Pi_{1}+B_{1}=\Pi_{1} S, \quad B_{1}=B L .
$$

A unique $\Pi_{1}$ always exists since the eigenvalues of $G_{1}$ and the imaginary eigenvalues in $S$ never add to zero. When the initial condition $x_{1}(0)=\Pi_{1} v(0)$ and input $u=a \cos (\omega t)$ the steady state solution of system is $x_{1}(t)=\Pi_{1} v(t)$. Therefore, the time argument in the steady state solution can be omitted without ambiguity; namely, $x_{1}=\Pi_{1} v$.

Set $x \otimes x=x^{(2)}$, because $x_{1}^{(2)}=\Pi_{1}^{(2)} v^{(2)}$ and $u$ is a scalar so $x_{1} u=x_{1} \otimes u=\Pi_{1} v \otimes L v=\left(\Pi_{1} \otimes L\right) v^{(2)}$. Equation (31) can be rewritten as

$$
C \dot{x}_{2}=G_{1} x_{2}+\left[\left(G_{2} \Pi_{1}^{(2)}+D_{1}\left(\Pi_{1} \otimes L\right)\right)\right] v^{(2)} \triangleq G_{1} x_{2}+B_{2} v^{(2)} \text {. }
$$

Moreover, the derivative of $v^{(2)}$ is

$$
\dot{v}^{(2)}=\dot{v} \otimes v+v \otimes \dot{v}=\left(I_{2} \otimes S+S \otimes I_{2}\right) v^{(2)} \triangleq S_{2} v^{(2)} .
$$

Equations (34) and (35) could construct another Sylvester equation like (23) with unknown $\Pi_{2} \in R^{n \times 4}$. The new Sylvester equation is

$$
G_{1} \Pi_{2}+B_{2}=\Pi_{2} S_{2}
$$

The steady state solution of (36) is $x_{2}=\Pi_{2} v^{2}$. Also $x_{1} \otimes$ $x_{2}=\left(\Pi_{1} \otimes \Pi_{2}\right) v^{3}, x_{2} u=\left(\Pi_{2} \otimes L\right) v^{3}$ and so on. Setting $B_{3}=G_{2}\left(\Pi_{1} \otimes \Pi_{2}+\Pi_{2} \otimes \Pi_{1}\right)+D_{1}\left(\Pi_{2} \otimes L\right)+D_{2} L \Pi_{1}^{(2)}, B_{3}=$ $G_{2}\left(\Pi_{1} \otimes \Pi_{2}+\Pi_{2} \otimes \Pi_{1}\right)+D_{1}\left(\Pi_{2} \otimes L\right)+D_{2} L \Pi_{1}^{2}$, the third Sylvester equation is

$$
G_{1} \Pi_{3}+B_{3}=\Pi_{3} S_{3} .
$$

The steady state solution of (37) is $x_{3}=\Pi_{3} v^{(3)}$.
Based on the analysis we could get the pattern of the steady state response $x_{k}=\Pi_{k} v^{\circledR}, \Pi_{k} \in R^{n \times 2^{k}}, k=1,2, \ldots$, and the steady state solution of system is

$$
x(t)=\sum_{n=1}^{\infty} x_{n}(t)=\Pi_{1} v(t)+\Pi_{2} v^{(2)}(t)+\Pi_{3} v^{(3)}(t)+\cdots .
$$

The initial condition $x(0)=\sum_{n=1}^{\infty} x_{n}(0)$. Equation (38) is exactly the same desired solution sought by SN algorithm.

In this Volterra algorithm because $\left\|v^{\circledR}\right\|=1,\left\|x_{k}(t)\right\|=$ $\left\|\Pi_{k} v^{\circledR}\right\| \leq\left\|\Pi_{k}\right\|$, the number of Sylvester equations has limitation. This algorithm could converge below any preset tolerance.

In order to simplify the algorithm programing, the traffic flow data is normalized as follows:

$$
X(n)=\frac{z(n)-\overline{z(n)}}{\operatorname{Max}[z(n)]-\operatorname{Max}[z(n)]},
$$

where $X(n)$ is the normalization time series, $z(n)$ is the original time series, $\overline{z(n)}$ is average value of original time series, and $\operatorname{Max}[z(n)]$ and $\operatorname{Min}[z(n)]$ are the maximum and minimum value of original time series.

\section{Simulation Results}

The effectiveness of the Volterra prediction algorithm is verified by the traffic flow shown in Figure 1. The 1500 data of 2500 traffic flow data are used to train the Volterra algorithm. The last 2 hours and 4 hours traffic data of November 27, 2013, are used to verify the effectiveness of the prediction algorithm mentioned in this paper. Also comparing with RBFNN prediction algorithm and old Volterra algorithm, the results show that the accuracy of new Volterra algorithm is better than RBFNN algorithm and old Volterra algorithm. The prediction error is shown in Table 1. The new Volterra 


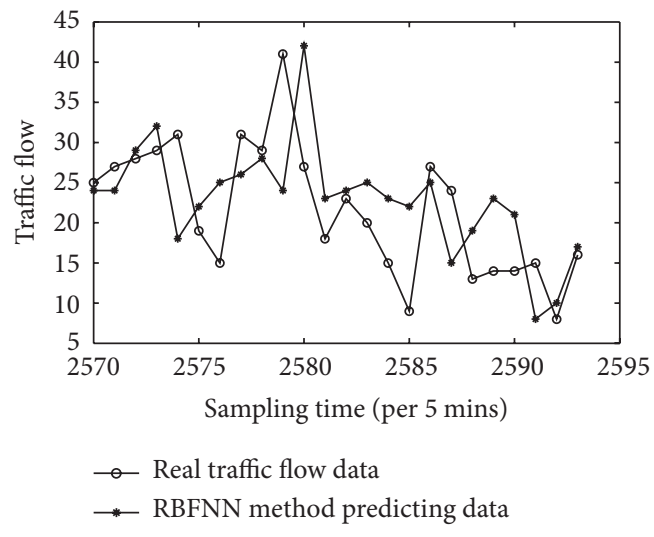

(a)

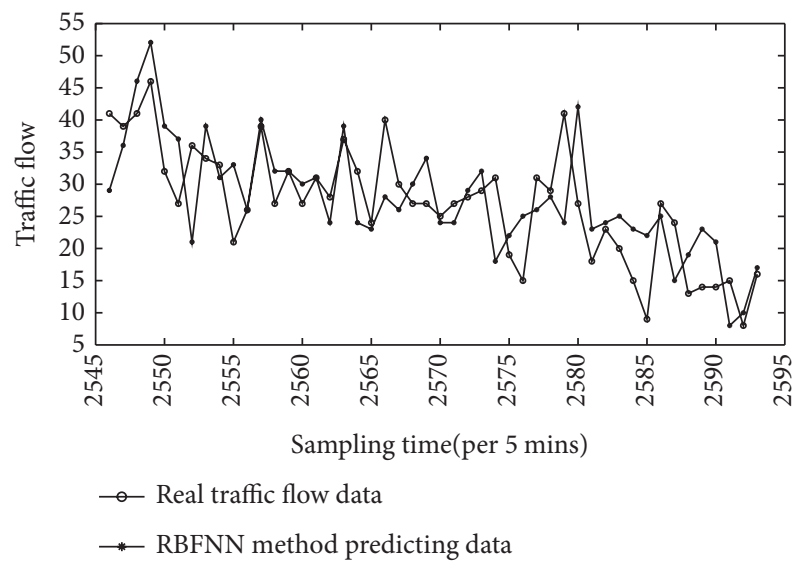

(b)

FIGURE 6: Prediction results of RBFNN algorithm: (a) 2 hours, (b) 4 hours.

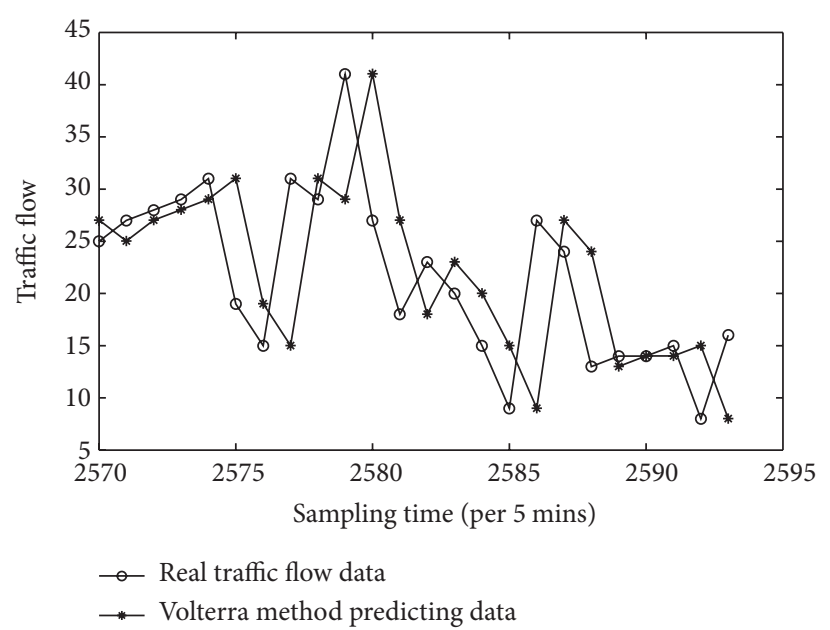

(a)

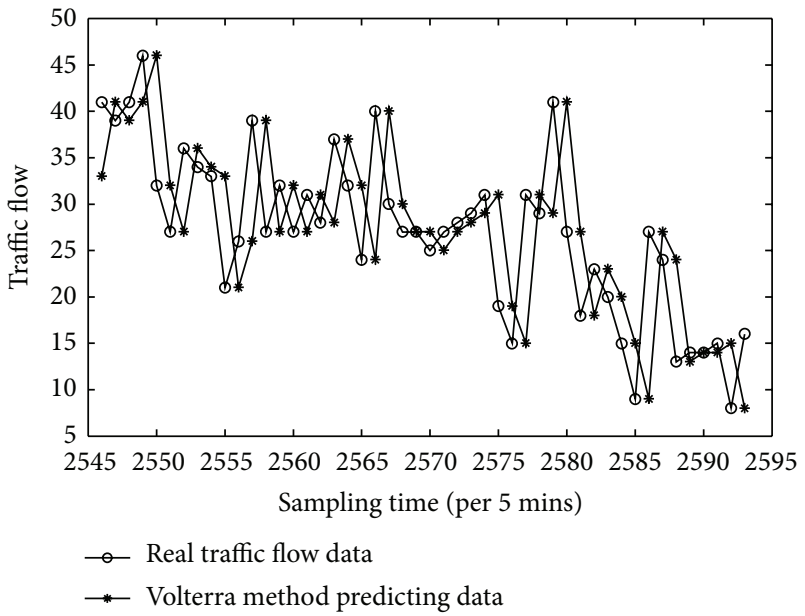

(b)

FIGURE 7: Prediction results of Volterra algorithm: (a) 2 hours, (b) 4 hours.

TABLE 1: Error of two algorithms.

\begin{tabular}{lcc}
\hline Model & 2h MAPE\% & 4h MAPE\% \\
\hline New Volterra algorithm & 3.98 & 7.2 \\
RBFNN algorithm & 5.21 & 10.04 \\
Volterra algorithm & 7.82 & 7.93 \\
\hline
\end{tabular}

algorithm prediction results are shown in Figure 5, and the RBFNN prediction results are shown in Figure 6; the old Volterra algorithm results are shown in Figure 7.

Through Figures 5, 6, and 7 it can be found that the trend of prediction is similar with real traffic flow. But the new Volterra algorithm is more accurate than RBFNN and old Volterra algorithm.

\section{Conclusion}

This paper analyzes the nonlinear character of Yu-Wu highway traffic flow time series. Using the largest Lyapunov exponent and correlation dimension to show that the traffic flow data are a chaotic time series, the traffic flow is reconstructed by phase space theory and the optimal time delay and embedding dimension are confirmed. Finally a new Volterra algorithm is used to predict the short-term traffic flow. The prediction results show that this algorithm could predict the short-term traffic flow effectively. The accuracy could satisfy the demand of highway guidance.

\section{Conflict of Interests}

The authors declare that there is no conflict of interests regarding the publication of this paper.

\section{Acknowledgments}

Project is supported by the National Nature Science Foundation of China (51407012) and Fundamental Research Funds for the Central Universities (2013G1321037). 


\section{References}

[1] D. Chunjiao, S. Chunfu, Z. Chengxiang, and M. Meng, "A shortterm state-space model for free flow prediction based on spatialtemporal characteristics," Tumu Gongcheng Xuebao, vol. 46, no. 8, pp. 111-118, 2013.

[2] H. Yin B, J. Xu M, and S. J. Huang, "Traffic flow prediction of signalized intersections using fuzzy_neural network approach," Journal of South China University of Technology, vol. 28, no. 6, pp. 11-15, 2000.

[3] X.-X. Weng and G.-L. Du, "Hybrid elman neural network model for short-term traffic prediction," in Proceedings of the 8th IASTED International Conference on Control and Applications, pp. 281-284, Montreal, Canada, May 2006.

[4] Z. Ye, Y. Zhang, and D. R. Middleton, "Unscented Kalman filter method for speed estimation using single loop detector data," Transportation Research Record, no. 1968, pp. 117-125, 2006.

[5] C. L. Yang, L. Jia, Q. L. He, and Q. J. Kong, "Study of traffic flow forecast algorithms based on chaotic wavelet networks," Journal of Shandong University, vol. 35, no. 2, pp. 46-49, 2005.

[6] Z. S. Yao, C. F. Shao, and Y. L. Gao, "Research on methods of short-term traffic forecasting based on support vector regression," Journal of Beijing Jiaotong University, vol. 30, no. 3, pp. 19-22, 2006.

[7] Y.-M. Zhang, X.-J. Wu, and S.-L. Bai, "Chaotic characteristic analysis for traffic flow series and DFPSOVF prediction model," Acta Physica Sinica, vol. 62, no. 19, Article ID 190509, 2013.

[8] S.-X. Liu, H.-Z. Guan, and H. Yan, "Chaotic behavior in the dynamical evolution of network traffic flow and its control," Acta Physica Sinica, vol. 61, no. 9, Article ID 090506, 2012.

[9] A. Wolf, J. B. Swift, H. L. Swinney, and J. A. Vastano, "Determining Lyapunov exponents from a time series," Physica D: Nonlinear Phenomena, vol. 16, no. 3, pp. 285-317, 1985.

[10] G. Barna and I. Tsuda, "A new method for computing Lyapunov exponents," Physics Letters A, vol. 175, no. 6, pp. 421-427, 1993.

[11] M. T. Rosenstein, J. J. Collins, and C. J. de Luca, "A practical method for calculating largest Lyapunov exponents from small data sets," Physica D, vol. 65, no. 1-2, pp. 117-134, 1993.

[12] B. H. Zhang, D. X. Sun, and Y. L. He, "Analysis and prediction of complex dynamical characteristics of short-term traffic flow," Acta Physica Sinica, vol. 63, no. 4, Article ID 040505, 2014.

[13] S.-Q. Zhang, J. Jia, M. Gao, and X. Han, "Study on the parameters determination for reconstructing phase-space in chaos time series," Acta Physica Sinica, vol. 59, no. 3, pp. 1576$1582,2010$.

[14] C. Gu, "QLMOR: a projection-based nonlinear model order reduction approach using quadratic-linear representation of nonlinear systems," IEEE Transactions on Computer-Aided Design of Integrated Circuits and Systems, vol. 30, no. 9, pp. 13071320, 2011. 


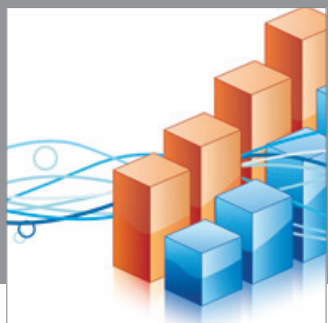

Advances in

Operations Research

mansans

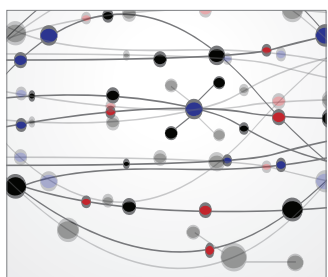

The Scientific World Journal
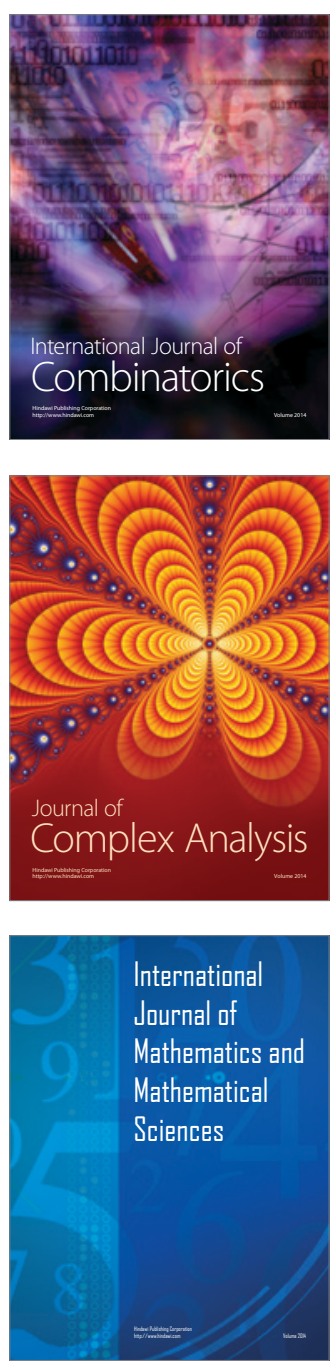
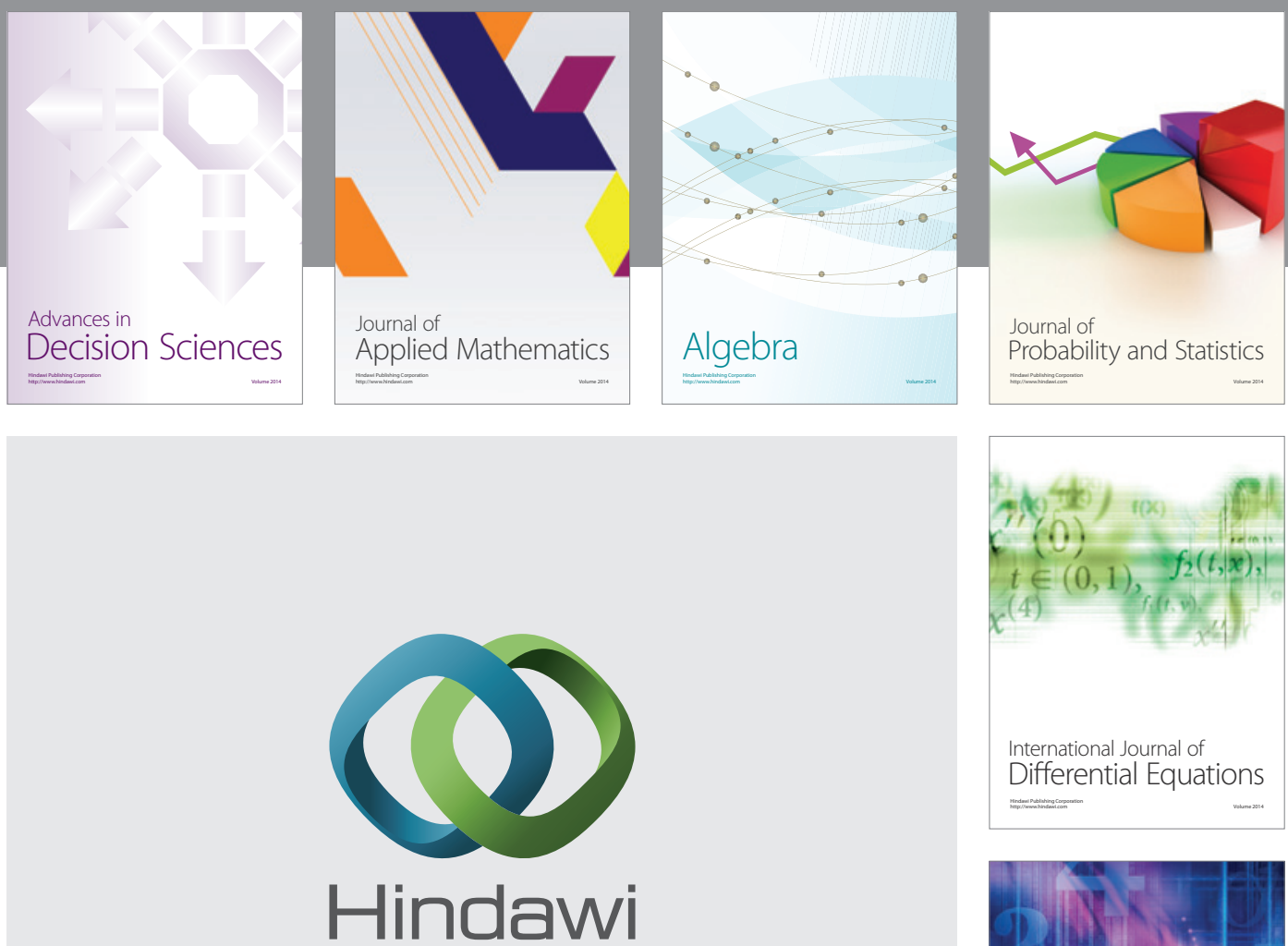

Submit your manuscripts at http://www.hindawi.com
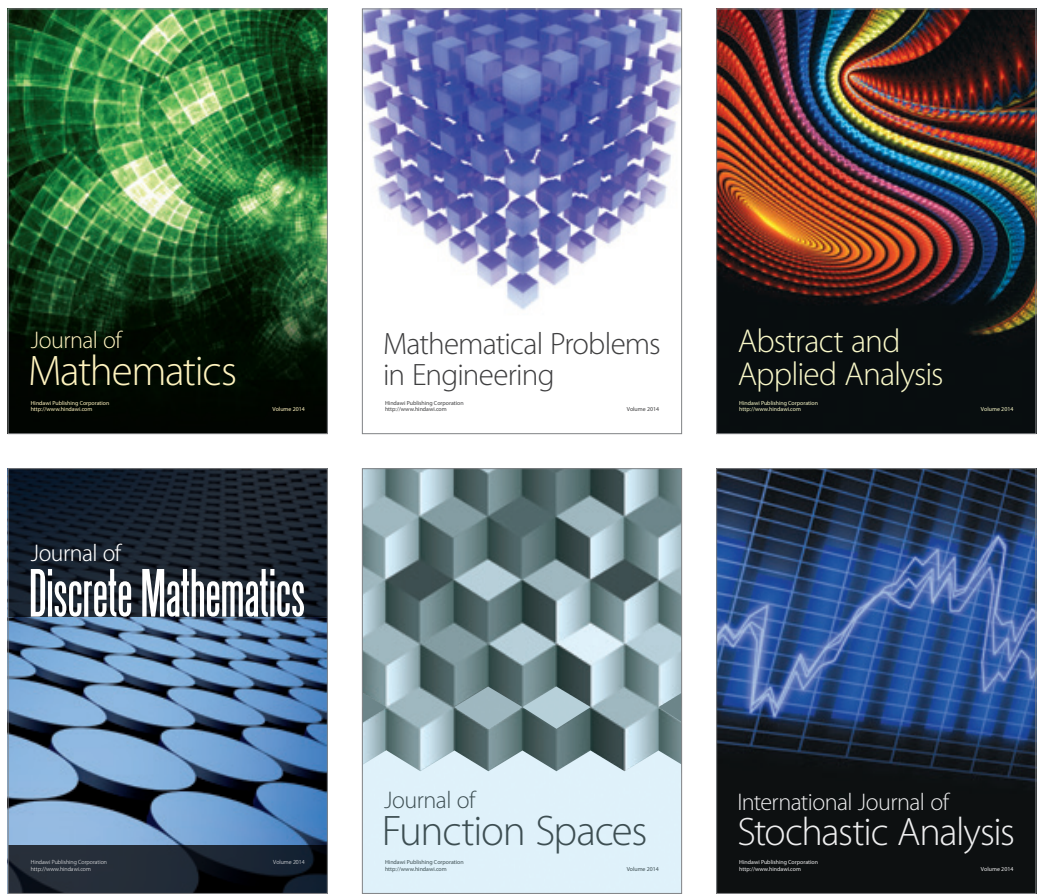

Journal of

Function Spaces

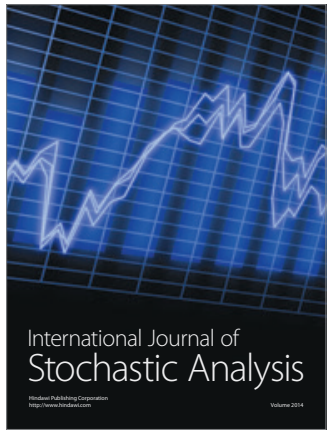

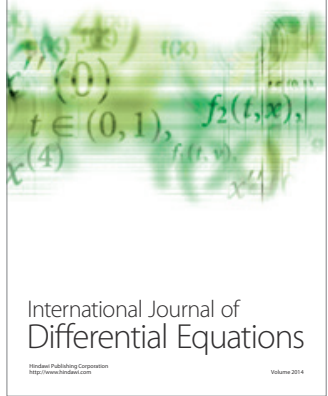
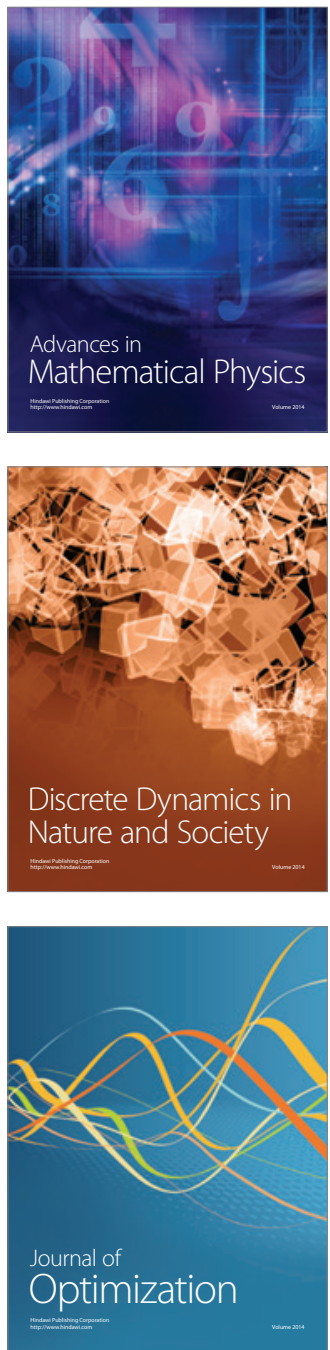